\title{
Integration of Optical and Wireless Technologies in the Metro-Access: QoS Support and Mobility Aspects
}

\author{
D. Remondo, M. Nunes, S. Sargento, M. Cesana, I. Filippini, J. Triay, A. Agustí, M. De Andrade, \\ Ll. Gutiérrez, S. Sallent, C. Cervelló-Pastor
}

\begin{abstract}
Future metropolitan and access networks are expected to comprise heterogeneous optical and broadband wireless technologies. The growing demand of users for transparent, ubiquitous access to diverse communication services poses several challenges. We envision a future metro-access architecture that comprises Optical Burst Switching networks that feed Ethernet Passive Optical Networks (PON) or upcoming Wavelength-Division Multiplexing PON, which in turn feed IEEE 802.16 and IEEE 802.11 nodes. Nodes in the wireless realm may communicate in a multihop fashion, forming mesh networks. To maintain cost and resource efficiency, we propose the introduction of Quality of Service $(\mathrm{QoS})$ proxies at the border between different link technologies. These entities handle QoS requirements and aid to the support of mobility. The architecture requires no modification of the Medium Access Control mechanisms of the different technologies.
\end{abstract}

Index Terms-Optical fiber communication, mobile communication, metropolitan area networks.

\section{INTRODUCTION}

$\mathrm{O}$ NE of the main problems of today's Internet is the performance bottleneck in the access segment. To address this issue, the great majority of researchers have proposed solutions to improve the efficiency of specific access technologies. However, we envision a network of the future where the user is able to access diverse services, in a transparent manner and with a high degree of ubiquity, while maintaining a high resource utilization efficiency. To comply with the expected future user's service demands, the network of the future will need to incorporate adequate access architectures that include mechanisms for the integration of different technologies.

Most of current access networks are based on copper

This work was partially supported by the Euro-NF Network of Excellence of the European Commission and the Spanish CICYT Project TSI200766637-C02-01, which is partially funded by FEDER.

D. Remondo, J. Triay, A. Agustí, M. De Andrade, Ll. Gutiérrez, S. Sallent and C. Cervelló are with Technical University of Catalonia (UPC), Barcelona, Spain (corresponding author: remondo@entel.upc.es).

M. Nunes is with INESC-ID, Lisboa, Portugal.

S. Sargento is with Institute of Telecommunications, Aveiro, Portugal.

M. Cesana and I. Filippini are with Politecnico di Milano, Milano, Italy. (mainly Digital Subscriber Lines and coaxial cables). The increasing communication service demand is making these networks obsolete and, in most cases, they are being gradually replaced by optical access networks. The most attractive optical access networks are Passive Optical Networks (PONs) [1], [2], which have no active internal elements and are thus excellent in terms of maintainability and robustness. The standardized PON architectures nowadays are based on TimeDivision Multiplexing (TDM), but the capacity per user-side terminal can be drastically augmented by means of Wavelength-Division Multiplexing (WDM).

Besides PONs in the access segment, the Optical Burst Switching (OBS) technology is seen as the next step in future optical networking in the metro segment [3]. In an OBS network, end-to-end optical paths are set up and torn down dynamically. The data that is transported in these optical paths forms thus data bursts. A separate control plane, in the electrical domain, is used to allocate the necessary resources in the optical domain. OBS networks [4] are expected to play an important role in future metropolitan networks, providing huge bandwidth capacities and easier interconnection to other WDM networks already being deployed in wide-area networks.

On the other hand, the attractiveness of ubiquitous access and the rapid progress in wireless technologies are making radio based communications more attractive for access networks. For broadband access, the most interesting options available nowadays are IEEE 802.16 [5] and IEEE 802.11 [6], which offer maximum transmission rates of several tenths of $\mathrm{Mbit} / \mathrm{s}$ at present. The upcoming amendments IEEE $802.16 \mathrm{~m}$ [7] and IEEE 802.11n [8] are expected to yield maximum user data rates above $100 \mathrm{Mbit} / \mathrm{s}$.

The literature contains some contributions to the integration of optical networks and wireless technologies in the metropolitan or access segments. One approach is to modulate radio signals onto optical frequencies, termed MicrowaveOver-Fibre (MOF) [9]. However, crosstalk among optical subcarriers represents a difficult issue that still needs to be solved.

The integration of metro and access networks is a new issue of research. The work of [10] presents a new hybrid 
architecture and a solution that copes with the integration of the backhaul optical and the wireless mesh network. It presents a solution for the integration based on reconfiguring the optical backhaul to balance the load among the different optical links in the backhaul side, and optimizing the routing from the end-user to the BS across the mesh nodes of the wireless network. Another recent work introduces a similar hybrid network architecture composed of optical backend and gateway-routers to connect the front-end users using either IEEE 802.11 or IEEE 802.16 devices [11]. The authors highlight the challenges in the deployment of such hybrid architecture and discuss station placement and routing issues in hybrid PON-wireless access networks. They have also presented updated versions of a routing algorithm to improve the network's efficiency. Although these two research works propose very interesting solutions in such scenarios, both approaches lack an analysis of the Quality of Service (QoS) aspects and the need to match the Medium Access Control (MAC) algorithms to optimize the overall network performance.

Progress in the design of future optical access networks is closely related to its integration with wireless technologies, as discussed in [12]. In fact, research directions point at architectures that contemplate WDM-PONs functioning as backhaul for IEEE 802.16 and IEEE 802.11n, where wireless links could form multihop wireless networks (mesh networks).

In this paper we introduce and comment on a hybrid optical/wireless access architecture for the support of present and future Internet applications. This work has been done in the context of the project Fi-Wi (Future Integrated Access Architecture Based on Optical and Broadband Wireless Technologies), a specific joint research project within the European Commission's Network of Excellence Euro-NF. Namely, we overview the enabling technologies that can be used to implement the hybrid network architecture, commenting on their potentials and effectiveness to support such network scenario. Technical issues rising from the integration of different technologies are critically discussed and research directions to achieve a fully functioning and optimally performing hybrid access architecture are provided.

In Section II we introduce the overall network architecture. Section III addresses the problem of supporting Quality of Service throughout the different segments of the hybrid network architecture. In Section IV, issues related to mobility management and support in the reference scenario are discussed, and finally, Section V concludes the paper.

\section{GLOBAL ARCHITECTURE OF THE NETWORK}

We envision future access networks that are formed by PONs that feed both IEEE 802.16 and IEEE 802.11 networks (see Fig. 1). Within a single PON, some of the Optical Network Units (ONU), which are each one of the edge nodes of the PON at the user's side, may be connected to an IEEE 802.16 base station (BS), and other ONUs may be connected to an IEEE 802.11 node. We do not exclude the possibility that IEEE 802.16 links can also be used to feed IEEE 802.11 nodes, given their relatively long communication range. The Optical Line Terminals (OLTs), which are the headend nodes of the PON, are connected to nodes in a metropolitan OBS network with a mesh topology.

Several types of single-wavelength PONs exist, but we consider Ethernet-PON (EPON, single wavelength networks based on IEEE 802.3ah) because it is the most cost-effective solution [1]. In view of future developments, we also consider upcoming WDM-PONs (a WDM-EPON standard is currently being developed by the IEEE 802.3av group).

Given the rapid evolution of standardisation in broadband wireless technologies, the IEEE 802.16 and IEEE 802.11 nodes can belong to different legacy or upcoming amendments. In the case of IEEE 802.16, they could be either IEEE $802.16 \mathrm{e}$ or IEEE $802.16 \mathrm{~d}$. In the former case, nodes can be mobile but can only form a point-to-multipoint (cellular) topology; in the latter case, nodes must be static, but multihop communication is possible within the IEEE 802.16 realm. The standard IEEE 802.16j allows static nodes to work as relay nodes, thus enabling multihop communication with the BS. Also, in the near future, IEEE $802.16 \mathrm{~m}$ compliant nodes could be included, with increased capacity.

For IEEE 802.11, the amendment IEEE 802.11n is of particular interest, since it provides aggregate user data rates in excess of $100 \mathrm{Mbit} / \mathrm{s}$ in the point-to-multipoint mode, allows for point-to-point (mesh) communication and incorporates traffic differentiation mechanisms for QoS support. Recent research work in advanced MAC mechanisms for multihop topologies shows that the achievable data rates are rather low due to fundamental limitations, namely the fact that interference ranges are in general much larger than transmission ranges. However, this holds only when interfering links use the same physical channel. If we make a proper assignment of the different physical channels available to the different links, the performance will be enhanced significantly. Therefore, the reference scenario supported in this paper considers two feasible architectures for the wireless extension part featuring a pure point-to-multipoint wireless topology and a real mesh topology where a multihop wireless network is integrated with the optical realm. 


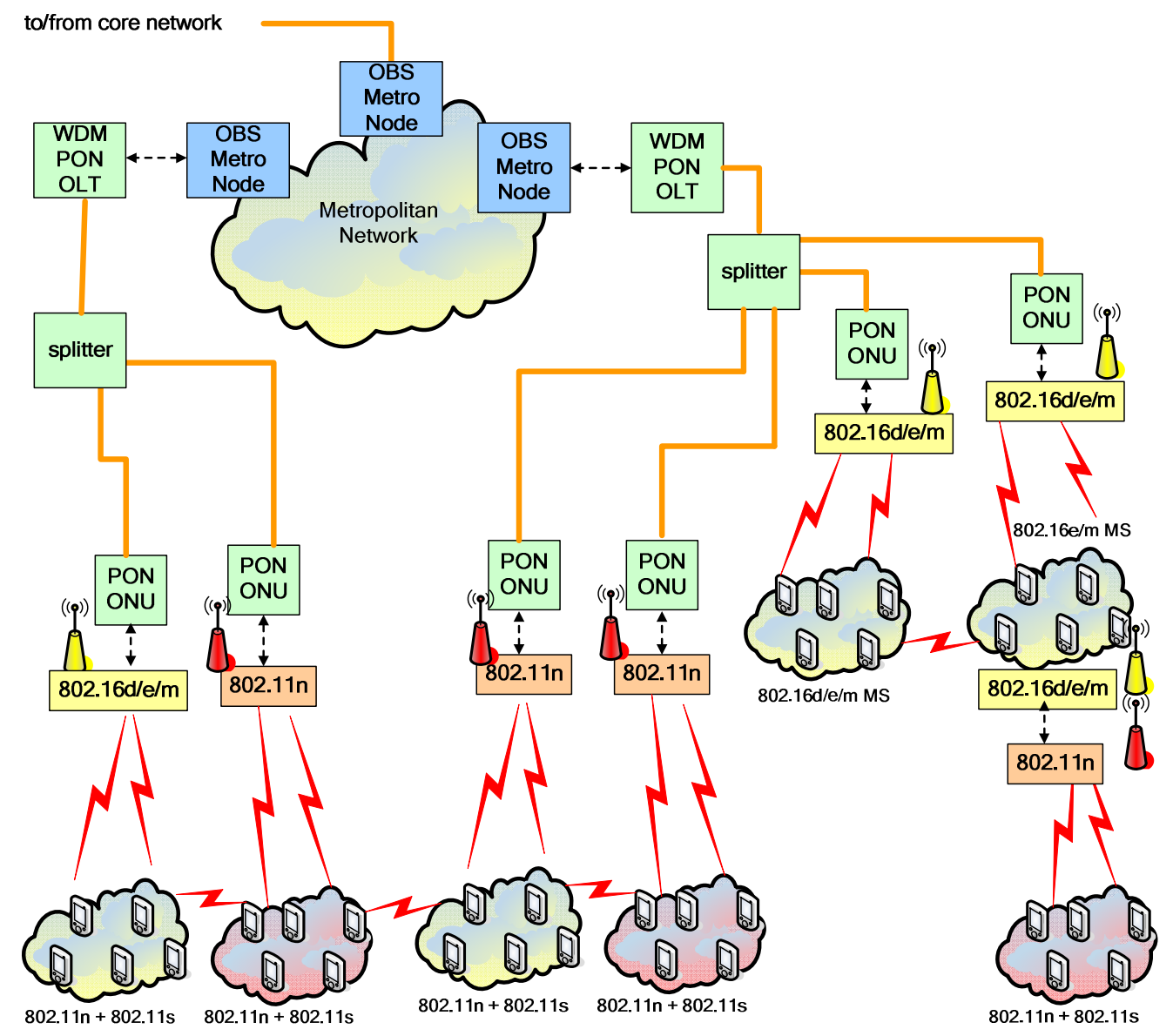

Fig. 1. Global architecture of the network

\section{QOS SUPPORT ARCHITECTURE}

Recent research work has been done to address the support of QoS in the specific scenario where IEEE 802.16 and EPON are integrated [13]. Every IEEE 802.16 BS is implemented into a single piece of equipment together with an ONU. To integrate the QoS support across the two realms, the authors describe three solutions: using MOF; replacing the Ethernet MAC layer in the PON by the IEEE 802.16 MAC layer; or a hybrid approach where the PON's MAC layer uses information on the connections established by the IEEE 802.16 MAC layer. The first solution presents physical limitations that are out of the scope of this work, while the second approach would not be suitable for PONs that comprise ONUs coupled with IEEE 802.16 and IEEE 802.11 nodes in the same network.

With the aim to support QoS in our scenario, we extend the hybrid approach of [13] to the integration of every couple of nodes that are on the border between two technologies. Between each couple of border nodes, we incorporate an entity that is in charge of the QoS support. This entity works as a QoS Proxy and does not imply modifications of the legacy MAC layers. The QoS Proxy operates above Layer 2 to take advantage of all the QoS mechanisms and functionalities defined in the MAC layer of each technology. A general view of the QoS architecture is shown in Fig. 2.

The main role of the QoS Proxy is to collect the resource requests received from the MAC entity of the segment downstream and forward them to the MAC entity of the segment upstream. To implement this functionality the QoS Proxy has to map the parameters that characterize the traffic QoS (i.e. priority classes, data flows, maximum sustained data rates per flow, maximum latency per flow, etc.) of the two technologies.

To maintain the scalability of the QoS architecture, the QoS requests sent by the different terminals below each QoS proxy should be aggregated before being sent upstream to the next level. This has a significant impact on the scalability and efficiency of the network, as the number of QoS requests and respective grants are significantly reduced.

When a new flow with QoS requirements is created, as well as when it is terminated, there is the need to inform the QoS Proxy involved. The specific operations involved in this process vary according to the signaling protocol, which is out of the scope of this work.

In the next sections we outline the functionality of the different QoS proxies that are needed, namely the QoS proxy between OBS and PON, the QoS proxy between the PON and 
the IEEE 802.16 BS and the QoS proxy between the PON and the IEEE 802.11n Access Point.

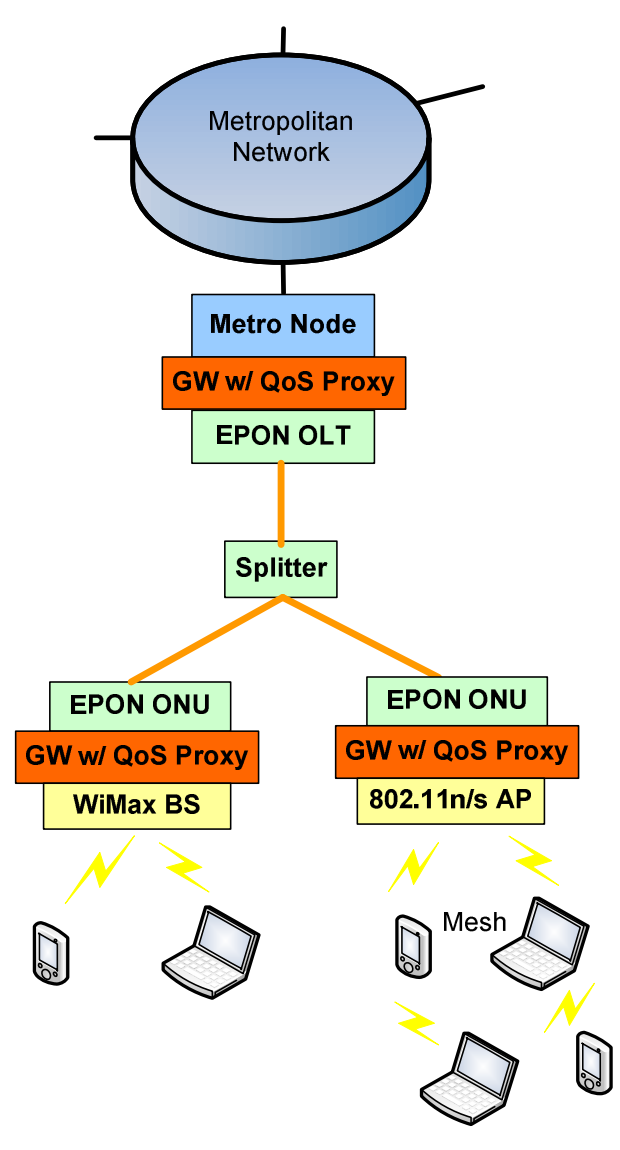

Fig. 2. General QoS architecture.

\section{A. QoS Support across $O B S$ and $P O N$}

A PON is a point to multipoint (P2MP) network with a functional tree topology. The OLT is connected to a passive optical splitter using an optical fibre, which fans out at the splitter to multiple optical drop fibers to which ONUs are connected. According to the standard IEEE 802.3ah, an EPON supports a nominal bit rate of $1 \mathrm{Gbit} / \mathrm{s}$, shared amongst the ONUs. In the downstream direction, the OLT transmits Ethernet frames that reach all the ONUs. In the upstream direction, the Ethernet frames transmitted by the ONUs are received only by the OLT. Medium access is arranged in a time-division fashion. The OLT arbitrates the upstream transmissions from the ONUs by granting transmission windows, which can have variable lengths, by means of GATE messages. ONUs use the transmission window to transmit the data along with REPORT messages to inform the OLT about its bandwidth requirements.

To prioritize traffic, the standard IEEE 802.3ah uses eight priority queues at the ONU for upstream traffic. The REPORT message actually contains information on the amount of data contained in the queues. Also, upon reception of a GATE message, the ONU is free to decide which data to transmit with an internal scheduler.

An extension to EPON is represented by WDM-PONs, where ONUs can be assigned multiple wavelengths (channels) each of which with a capacity of $1 \mathrm{Gbit} / \mathrm{s}$ (or even extended to $10 \mathrm{Gbit} / \mathrm{s}$ ). The wavelength assignment algorithms/policies are not standardized and can be optimized by the designer/vendor. The migration and simultaneous operation of PON and WDMPON must be transparent.

Regarding OBS, two operational planes are defined: the data plane, which is all-optical, and the control plane, in which control packets are processed electronically (although they can be transported optically). These control packets carry out the task of reserving optical resources. The optical burst is the basic data unit of OBS and is composed of several shorter packets that have the same destination node or the same QoS treatment. Traditionally, the transmission of the optical burst is done after an offset time, during which the control packet tries to reserve the resources along the path from the origin OBS node to the destination OBS node. Often, OBS uses oneway reservation protocols (without positive acknowledgement) and, as a consequence, collisions in the network might occur.

In OBS networks, QoS can be provided using different approaches: (1) by providing an expected burst blocking probability through variable time offsets or running certain Routing and Wavelength Assignment (RWA) protocols [14], (2) controlling the desired end-to-end delay which is highly influenced by the burst assembly process [15], or (3) using different classes of reservation schemes [16].

To maintain QoS requirements, the OBS-PON QoS proxy needs to monitor or even control some of the input traffic characteristics. For this purpose, we need to establish a signaling protocol between this QoS proxy and the downstream QoS proxies. On the one hand, the upstream gateway needs information from the downstream QoS proxy on the destination and QoS parameters of the upstream traffic arriving from the wireless networks. On the other hand, the OBS-PON QoS proxy might have to ask each ONU what traffic should be granted at each cycle for being transmitted.

It is not an objective of this signaling protocol to extensively modify the current protocol standardizations, therefore signaling should run in accordance to the standards involved in the architecture and be backward compatible with the IEEE 802.3ah standard [17] or future WDM-PON. However, to meet our architecture requirements some extra functions will need to be added to the reporting and gating processes of the OLT and ONUs. In this paper we propose to use the padding or reserved bits of the REPORT and GATE PON messages for signaling purposes. This signaling protocol will support the QoS proxy entities, allowing to define a more global and efficient QoS management at the OBS-PON QoS proxy, without the need for additional transmission resources.

Both the REPORT and GATE messages exchanged between the OLT and the ONU, as described in the standard 
IEEE 802.3ah, contain a certain amount of $\mathrm{pad} / \mathrm{reserved} \mathrm{bits.}$ We propose the use of these bits for the following purpose. In the REPORT message, the extra bytes are used to notify the OBS-PON QoS proxy about the destination of the packets currently stored in the priority queues. Similarly, in the GATE message, the extra bytes are used to notify the wireless-PON QoS proxy about the destination and priority/class of service of the frames that should preferably be transmitted in the next granted period. In order to allocate as much data as possible in each OBS burst, we need a mechanism to map IP addresses to possible destinations in the OBS-PON network. As we do not expect the metro network to have many nodes that need to be identified (i.e. those nodes directly connected to core routers or to access networks), the mapping table sizes will not be very large. Therefore, 8 bits would suffice to designate all the possible destinations ( 5 bits) and priorities/classes of service (3 bits).

\section{B. QoS Support across PON and IEEE 802.16}

In IEEE 802.16, Service Data Units (SDUs) are associated with the proper service flow identified by a Connection Identifier (CID). A service flow is a unidirectional flow of packets that is provided with a particular QoS. In the point-tomultipoint mode, a MAC-level request-and-grant mechanism between Subscriber Stations (SSs) and BS is used to accommodate the dynamic demand of service flows. An SS requests the amount of resources needed for each connection and the BS grants resources for all the SS's connections as a whole. The request-and-grant mechanism is fitted into $5 \mathrm{~ms}$ long Time-Division Duplex (TDD) frames.

In E-PON, every ONU classifies upstream packets into eight priority queues and sends information on its queue sizes to the OLT. The OLT assigns a certain amount of resources to each ONU and the ONU assigns the resources to its queues with an internal scheduler. However, when the ONU is integrated with an IEEE 802.16 BS, we could gain a lot of control over the end-to-end QoS if the detailed and finegrained (per connection) knowledge that is available at the IEEE 802.16 BS could be employed in the PON segment and even in the metro OBS segment. Not only has the IEEE 802.16 BS the most complete information on the users' upstream traffic needs, but in principle it distributes the resources in the most appropriate manner to comply with the Service Level Agreements (SLAs), while taking into account the variable nature of the wireless channel bandwidth [5]. Therefore, we have to look at a mechanism that relates the distribution of resources made by the BS to the assignment of resources within the PON and metro OBS segments.

In conclusion, the QoS proxy between the ONU and the IEEE 802.16 BS has the following functionality:

- Implement a co-operative admission control mechanism with the QoS proxy in the upstream direction. The ONU-IEEE 802.16 proxy sends information about the connections that the BS wants to establish with its associated SSs to the upstream proxy and tells the BS whether to admit the new connection depending on the response received.

- Exchange signaling messages with the upstream QoS proxy in order to provide information about the destination of the packets currently stored in the ONU's priority queues, as mentioned in the previous section.

- Instruct the internal ONU scheduler to select packets for upstream trying to comply with the GATE messages received from the upstream proxy, while taking into account the QoS parameters and amount of incoming traffic for each upstream connection or set of connections in the IEEE 802.16 BS.

The last of these three functionalities of the QoS proxy is probably the most challenging part of the QoS architecture, since the proxy has to comply with the demands from the upstream proxy, which would like to receive packets with the same destination from all ONUs consecutively, while the SLAs negotiated between the BS and its SSs per connection should be maintained. Specific solutions will be investigated in follow up of this work.

\section{QoS Support across PON and IEEE 802.11}

The IEEE 802.11 Access Point that is integrated with the ONU may be configured to work either in infrastructure mode (point-to-multipoint topology) or to function as a gateway of a mesh network topology. In both cases, to support QoS we need the wireless technology to provide traffic differentiation mechanisms. At the present technological context of IEEE 802.11 , this can only be done with the amendments IEEE 802.11e or IEEE 802.11n. A basic feature for QoS support is to map classes between the wireless realm and the ONU. Moreover, in the case of IEEE 802.11n, the impact of frame aggregation on the upstream traffic characteristics should be taken into account.

When wireless multihop topologies are considered, the issue of managing and enforcing QoS on the wireless segment of the overall access network requires research at different layers of the protocol stack. Indeed, several features have a strong impact on the performance of a general Wireless Mesh Network (WMN), including the number of radio interfaces for each device, the number of available radio channels, the medium access mechanism, the routing strategies and the specific wireless technology used to implement the mesh paradigm. As a consequence, in order to enable any kind of QoS mechanism in the multihop wireless realm, we need to establish routes in this segment through specific strategies and to tune the MAC specifically for this context.

Moreover, since network deployments can involve hundreds of devices, manual tuning and reconfiguration are very unpractical and the automatic planning and optimization of the network coverage and topology is of utmost importance to efficiently provide the required services. To this extent, optimization models and tools are required to manage the coverage and topology planning of the hybrid optical/WMN 
architecture, by making wise decisions on parameters like the positions of ONU/802.11 gateways, the number of radio interfaces to be used by wireless devices and the channels to be used on each wireless link.

Even if the deployment optimization process tends to reduce the interference per wireless link, still co-channel interference may affect the quality of the communications. To this extent, effective mechanisms/approaches to cope with interference and manage the channel access are needed, after deploying/optimizing the wireless topology.

As an example, if we consider a IEEE 802.11s [18] based WMN, with Mesh Deterministic Access (MDA) [19], [20] as the medium access scheme, all transmissions start at the preagreed time and end successfully. However, the available bandwidth is reduced by the use of beacons to co-ordinate medium access. IEEE $802.11 \mathrm{~s}$ defines a WMN wide parameter called MDA Access Fraction (MAF) that is the fraction of the beacon period that a mesh router can use for MDA access. The choice of MAF has an enormous effect on the performance of a $802.11 \mathrm{~s}$ WMN since a small MAF value leads to a situation in which legacy 802.11 devices have a large share of the beacon period, so can potentially generate more traffic that can be relayed by the mesh network. On the other hand, high MAF values lead to a situation in which mesh routers are competing to send data, since mesh routers occupy almost all the super-frame, thus throttling legacy 802.11 devices access fraction. This value defines the maximum theoretical bandwidth available in wireless mesh networks, and is given by

$$
M A F=G /(1+G),
$$

where $G$ is the MDA time. The previous formula shows that when there are many mesh routers, i.e. a low fraction is dedicated to serve a stream from a given mesh router, then the MAF value approaches one; this is logical, since in this case it is better to attribute a very large of the beacon period to MDA access, as nodes are transmitting to their Access Points at a very low rate.

The above description considered a specific case of MDA access in single-channel WMNs. However, the problem of unpredictability of the bandwidth available will be in place in any wireless mesh network. We must take into account that wireless nodes may be equipped with multiple interfaces and different wireless links may use different physical channels as well. Even if we use MDA on a single channel, the determined value for the available bandwidth is used for the overall network without differentiating paths. On the other side, the provision of knowledge of the different conditions in different parts of the network will decrease scalability of the QoS architecture, as this would require complex admission control mechanisms in the QoS proxy. It is then required to specify a QoS mechanism for single and multiple channel wireless mesh networks that is able to infer the QoS characteristics of the wireless mesh network. This mechanism will then be integrated in the QoS proxy, to be able to support QoS oriented admission control and bandwidth allocation efficiently and with good scaling properties, through the inference mechanisms.

\section{MOBILITY SUPPORT ARCHITECTURE}

The support of mobility in this architecture is not a trivial process. The architecture contains different types of integrated networks, where users may be connected to different technologies, IEEE 802.11 or IEEE 802.16 , possibly in a mesh topology, and integrated with IEEE 802.16 backhaul and optical core. In this sense, we need to provide mobility of the users in each possible scenario, mobility inside the mesh network, between networks in different IEEE 802.16 backhaul, and between networks in different PONs.

Mobility management consists of two important tasks: location and handoff management. Location management handles location registration and data delivery, while handoff management is responsible for handoff initiation, new connection generation, and data flow control for session handoff. Considering that we are dealing with the most complex case of the users in a mesh network, handoff is indispensable for connection continuity, as a user moves from the range of one mesh router to that of another. Ideally, the handoff should be completely transparent to users, characterized by low packet loss, minimal handover latency, low signaling traffic overhead, limited handover failure and the similarity of QoS provided by the source and target systems.

In a seamless mobility scheme for users possibly connected to wireless mesh networks in heterogeneous scenarios, some requirements need to be taken into account:

- Users should not require special configuration and should be able to work in any network, to support the above envisioned scenario.

- Inherent characteristics of wireless mesh networks need to be handled: multi-hop and multi-path routing, multi-interface and multi-channel.

- The handoff should be prepared before it occurs (make-before-break).

- Cross-layer mechanisms between all the layers involved in the mobility process should be used to reduce the handover time.

- The information about the mesh routers and users, including prediction and provision of the user's movement should be distributed across the mesh network.

- Separation of entities and addresses of users requires maintaining the mapping of this information in a distributed manner should be in place.

- Heterogeneity in terms of wireless access technologies, services, application requirements, devices capabilities, high usability and improved capacity should be taken into account.

- To globally increase the scalability of a mobility 
scheme, an efficient gateway assignment scheme is needed (incorporating macro-mobility). In this case a WMN can be viewed as an entire access point.

In such scenarios, the well-known protocols to mobile adhoc networks may not be efficient to directly apply in these networks, since they do not consider their stationary property and heterogeneity of networks. Also, due to the diversity of wireless mesh networks, it may not be so efficient to directly apply a centralized database for mobility management (as in the cellular networks).

Some recent mobility management proposals try to integrate some aspects related before. Most of them find a simple way to perform a seamless L2 handover (using gratuitous ARP messages, make-before-break models aided by mobile agents) and to decrease the L3 post-handover optimization time (with tunnel- or routing-based solutions, or with geographical routing solutions). The existing proposals are network-based, so, there is the need to create, distribute, update and locate the context-information of the network and users (mapping between users and APs and AP's neighboring tables/databases) with low signaling, low overhead and low cost. Most of the proposals separate the address and the identifier of a user, or create a MAC-to-IP mapping addressing in order to retain the user's IP address after handoff (one of drawbacks of Mobile IP-based [21] solutions is the need for Care-of-Addresses).

For the support of this heterogeneous scenario, there is the need to find a solution for global mobility (this is, between networks). For this support, there is the need of an efficient gateway assignment for a specific user, which is a topic studied by [22], [23], [24]. Rather than proposing only an efficient algorithm for gateway assignment, which tries to balance the traffic between the various existing gateways, allowing multi-homing, it is required to integrate mobility of end-users between different wireless mesh networks with the gateway assignment problem, taking into account the context of the user and the network. Some other proposals try to incorporate global mobility in their solutions but they require Mobile IP as an intermediate [25] [26] [27]. We will then need to support the efficient integration of a local and global mobility mechanism with seamless support of different networks, able to integrate with backhaul wireless links and the optical realm.

The approach envisioned considers the division of mobility in global and local mobility levels, and is based on a mobilespecific routing approach allied with a purpose-built IPv6 addressing scheme. This scheme was devised in order to easily identify the mobile terminal and the access network in which it currently is (the mobile terminal's Local Address identifies both the mobile terminal and its access network), which, coupled with the wireless mesh routing protocol, solves most of the issues with the local mobility. At the global mobility level, an IPv6 mapping table is kept to maintain the association between the local mobile terminal address and its purpose-built global address. For the outside, the mobile terminal always keeps the same IPv6 address (the global one), thus masking its movement, while internally the mobile node's address changes according to its position.

\section{V.CONCLUSION}

The presented architecture has been defined according to the expected future evolution of broadband wireless networks and optical access and metropolitan networks that will be soon available with upcoming optical technology.

To this extent, we have commented on the research challenges that kick in the hybrid wireless/optical access segment, with a particular focus on QoS support and users mobility management.

We have proposed the use of QoS proxies, which can handle QoS across the network and facilitate mobility support in the wireless realm so that no modification of the existing MAC mechanisms is required. This is a highly scalable solution, since adjacent QoS proxies exchange signaling information, thereby allowing fine admission control and providing useful information to the data schedulers in the different network nodes for handling heterogeneous services.

Future work will concentrate on the research roadmap discussed in the paper including: the development of signaling infrastructures/protocols among QoS proxies, the design of new schedulers to handle different types of service in the different segments, the optimization of the network topology considering the optical and the wireless mesh realms together, and the integration of mobility support with gateway assignment without resorting to Mobile IP in a metropolitan/access wide context.

\section{REFERENCES}

[1] G. Kramer, Ethernet Passive Optical Networks, Mc Graw-Hill Professional Engineering, 2005.

[2] T. Koonen, "Fiber to the Home/Fiber to the Premises: What, Where, and When?," Proc. IEEE, Vol. 94, No. 5, May 2006.

[3] M. O'Mahony, C. Politi, D. Klonidis, R. Nejabati and D. Simeonidou, "Future Optical Networks," J. Lightw. Technol., vol. 24, no. 12, Dec. 2006.

[4] Y. Chen, C. Qiao and X. Yu, "Optical Burst Switching: a new area in optical networking research", IEEE Network, vol. 18, num. 3, pp. 16-23, May 2004.

[5] C. Hoymann, "Analysis and performance evaluation of the OFDM-based metropolitan area network IEEE 802.16," Computer Networks, 49 (2005), Elsevier.

[6] IEEE 802.11 WG, Part 11: Wireless LAN Medium Access Control (MAC) and Physical Layer (PHY) Specification, 1999.

[7] http://wirelessman.org/tgm/docs/80216m-08_003r5.zip

[8] Y. Xiao, "IEEE 802.11n: Enhancements for Higher Throughput in Wireless LANs," IEEE Wireless Communications, Dec. 2005.

[9] A. Nirmalathas et al., "Wavelength Reuse in the WDM Optical Interface of a Millimetre-Wave Wireless-Wireless Antenna Base Station," IEEE Trans. Microwave Theory, vol. 49, no. 10, Oct. 2001. 
[10] W.-T. Shaw, S.-W. Wong, N. Cheng, K. Balasubramanian, X. Zhu, M. Maier, L.G. Kazovsky, "Hybrid Architecture and Integrated Routing in a Scalable Optical-Wireless Access Network," J. Lightw. Technol., vol. 25, no. 11, Nov. 2007.

[11] S. Sarkar, S. Dixit, and B. Mukherjee, "Hybrid Wireless-Optical Broadband Access Network (WOBAN): A Review of Relevant Challenges," J. Lightw. Technol., vol. 25, no. 11, Nov. 2007.

[12] L.G. Kazovsky, W.-T. Shaw, D. Gutiérrez, N. Cheng, S.-W. Wong, "Next-Generation Optical Access Networks," J. Lightw. Technol., vol. 25, no. 11, Nov. 2007.

[13] G. Shen, R.S. Tucker and C.-J. Chae, "Fixed Mobile Convergence Architectures for Broadband Access: Integration of EPON and WiMAX," IEEE Commun. Mag., Aug. 2007.

[14] J. Teng and G.N. Rouskas, "Wavelength Selection in OBS Networks Using Traffic Engineering and Priority-Based Concepts," IEEE JSAC, vol. 23, no. 8, Aug. 2005.

[15] P. Reviriego, J.A. Hernández and J. Aracil, "Analysis of average burstassembly delay and applications in proportional service differentiation", Photonic Network Communications, vol. 14, num. 2, pp. 183-197, October 2007.

[16] K. Vlachos and K. Ramantas, "A non-competing hybrid optical burst switch architecture for QoS differentiation", Optical Switching and Networking, vol. 5, no. 4, pp. 177-197, October 2008.

[17] Standard 802.3-2005, IEEE.

[18] Joint SEE-Mesh/Wi-Mesh Proposal, doc.: IEEE 802.11-06/0329r3, March 2006.

[19] S. Kangude and H. Chhaya, Mesh Deterministic Access, US Patent United States Patent 20070060141.

[20] C. Cicconetti, L. Lenzini, E. Mingozzi, "Sensor, Scheduling and Dynamic Relocation for IEEE 802.11s Mesh Deterministic Access", 5th Annual IEEE Communications Society Conference on Mesh and Ad Hoc Communications and Networks, SECON, June 2008.

[21] C. E. Perkins, IP Mobility Support for IPv4, RFC 3344, Aug., 2002.

[22] M. Ren, C. Liu, H. Zhao, T. Zhao, W. Yan, "MEMO: An Applied Wireless Mesh Network with Client Support and Mobility Management," IEEE Global Telecommunications Conference (GLOBECOM 2007), Nov. 26-30, 2007.

[23] E. Bortnikov, I. Cidon, I. Keidar, "Scalable Real-time Gateway Assignment in Mobile Mesh Networks," CoNEXT'07, New York, U.S.A, Dec. 10-13, 2007.

[24] R.Baumann, O.Bondareva, S. Heimlicher, M. May, "A Protocol for Macro Mobility and Multihoming Notification in Wireless Mesh Networks", $21^{\text {st }}$ Int. Conference on Advanced Information Networking and Applications Workshops (AINAW 2007), 2007.

[25] A. Pathak, A. Srivatsa, J. Xie, "An Analytical Model for Handoff Overhead Analysis in Internet-based Infrastructure Mesh Networks," Proc. IEEE Int. Conference on Communications (ICC 2008), pp. 2884-2888, Beijing, China, May 19-23, 2008.

[26] S. Sabeur, G. Al Sukkar, B. Jouaber, D. Zeghlache, H. Afifi, "Mobile Party: A Mobility Management Solution for Wireless Mesh Network", $3^{\text {rd }}$ Int. Conference on Wireless and Mobile Computing, Networking and Communications (WiMob 2007), Oct. 8-10, 2007.
[27] B. Xie, Y. Yu, A. Kumar, D. Agrawal, "Load-balancing and Interdomain Mobility for Wireless Mesh Networks", IEEE Global Telecommunications Conference (GLOBECOM 2006), 2006. 